\title{
Zwei Überlegungen zur Konzeption einer Linked-Data-Ontologie für die Literaturwissen- schaften
}

\author{
Bernhard Oberreither
}

ÖAW, ACE - Austrian Corpora and Editions

bernhard.oberreither@oeaw.ac.at

Keywords: Linked Open Data, Semantic Web, Literary Studies, Intertextuality, Ontology

\begin{abstract}
The paper outlines reflections on constructing a Linked Data ontology for literary studies, especially intertextuality. In particular, a case is made for two modeling principles - textualism and implicitness of methodological specifics -, which are briefly discussed with regard to different demands to DH made by proponents as well as critics of this field of research.
\end{abstract}

Vorbemerkung: Im Folgenden werden - in aller Kürze - Überlegungen vorgestellt, die der Konzeption der Ontologie INTRO („Intertextual Relationships Ontology“) vorausgingen. Die Ausführungen gehen auf einen Vortrag auf der dha2018 zurück, beschränken sich dabei jedoch auf einen Teil der damals behandelten Inhalte. Einen Überblick über INTRO selbst sowie Beispielmodellierungen finden sich im readme auf GitHub. ${ }^{1}$

I.

For nearly six centuries, humanistic models of knowledge have been shaped by the power of print as the primary medium of knowledge production and dissemination. Rather than rejecting print culture or embracing the simple pouring of print models into digital molds, Digital Humanities is engaged in developing print-plus and post-print models of knowledge. Both involve more than an updating of the knowledge delivery system. They entail the cognitive and epistemological reshaping of humanistic fields as a function of the affordances provided by the digital with respect to print. ${ }^{2}$

- so eine Definition der digitalen Geisteswissenschaften in der jüngeren Einführungsliteratur. Digitale Geisteswissenschaften, kann man dem entnehmen, seien kein bloßes Update, vielmehr eine kognitive und epistemologische Neu- oder Umgestaltung der Geisteswissenschaften, und gehen also, wie sich daraus schließen lässt, weit über den Einsatz von Textverarbeitungs-Programmen und die Gestaltung von informativen Projekt-Websites hinaus. Passagen wie diese sind natürlich normativ mehr denn deskriptiv, oder, anders ausgedrückt: Beschwörungen eher als Beschreibungen. Wer in den digitalen

1 Vgl. Bernhard OвERREITHER, INTRO. An Intertextual Relationships Ontology for literary studies, online unter: https://github. com/BOberreither/INTRO (16.04.2019).

2 Ann Burdick u. a., Digital_Humanities, Cambridge (Mass.) / London 2012, 125. 
Geisteswissenschaften publiziert, landet bald in diesem Register, das gilt natürlich auch für den Bereich Linked Data (und schließt den vorliegenden Text mit ein). Zu bedenken ist dabei, dass des einen Wunschvorstellung des anderen Schreckensvision sein kann. In seiner schon aufgrund ihres hohen Unterhaltungsfaktors sehr lesenswerten Breitseite gegen die Digital Humanities und in direkter Bezugnahme auf die oben zitierte Publikation schreibt Jeremy Brennan 2017:

The authors summarize the intellectual activities they promote: "digitization, classification, description and metadata, organization, and navigation." An amazing list, which leaves out that contradictory and negating quality of what is normally called "thinking". It would be a mistake to see this banishment of the concept as the passive by-product of a technical constraint. It is the aim of the entire operation. ${ }^{3}$

Das Denken gehe am Übergang zu den digitalen Geisteswissenschaften verloren, und abseits der Polemik kann man dem immerhin soweit folgen, dass Textarbeit bei der Anwendung etwa von text mining, topic modeling etc. nicht mehr primär der humanen agency obliegt. Der Fluchtpunkt solcher und ähnlicher Einwürfe liegt wohl in der Frage: Angenommen, Kulturwissenschaft ist von ihrem Gegenstand nicht getrennt, sondern tatsächlich Teil von Kultur, angenommen außerdem, dass Menschen die zentralen Akteure von Kultur sind - welchen Stellenwert kann dann ein Ergebnis beanspruchen, das nicht-humaner agency entspringt?

Auf der anderen Seite stehen Vertreter*innen der digitalen Geisteswissenschaft, die mit Recht auf einige Unzulänglichkeiten ihres traditionell arbeitenden Gegenübers hinweisen: auf die oft mangelhafte Nachvollziehbarkeit der Datenverarbeitung (also des Denkens), die oftmals geringe Datengrundlage, und, seit einiger Zeit, das, was als ,Datensilo-Problematik' bekannt ist: Forschungsergebnisse in traditionellen Publikationsformen sind (das betrifft auch digitale Formate wie Datenbanken etc.) meist nicht standardisiert, somit nicht automatisiert mit weiteren Ressourcen zu verknüpfen, und damit einer vielversprechenden Möglichkeit - nämlich der Datenakkumulation und der Abfrage aus kombinierten Datensets - beraubt. „Set your Data free!“ lautet dementsprechend der Impetus der Vertreter*innen von Linked Data, ebenso der Titel eines entsprechenden Vortrags aus dem Jahr $2017 .{ }^{4}$

Das Potenzial von Linked Data ist tatsächlich groß: So lässt sich mit dieser Technologie der Datensilo-Problematik begegnen, indem einerseits Ergebnisse maschinenlesbar und automatisiert verknüpfbar gemacht werden, und indem andererseits die oft in puncto Vorwissen voraussetzungsreichen Aussagen aus den verschiedenen Disziplinen auch an eben dieses Vorwissen, an ihre methodischen oder theoretischen Grundlagen rückgebunden werden können (um den Datensilo so ein zweites Mal zu durchstoßen, nun gewissermaßen vertikal). ${ }^{5}$

Für Datenintegration dieser Art greift Linked Data auf standardisierte Metadaten-Vokabulare zurück, die jeweils einen bestimmten Ausschnitt der Welt abbilden. Vokabulare dieser Art, sog. ,Ontologien“, können mittels eines zugleich hierarchisch und über Relationen strukturierten Klassensystems etwa auch geistes- bzw. literaturwissenschaftliche Leistungen im Semantic Web darstellen.

3 Timothy Brennan, The Digital-Humanities Bust. After a decade of investment and hype, what has the field accomplished? Not much, in: The Chronicle of Higher Education, 15.10.2017.

4 Vgl. Torsten SCHRADE, „Set your data free“ - Vom geisteswissenschaftlichen Datensilo zur vernetzten Wissensbasis mittels Linked Open Data. Siehe Martin PreLL, Digital Humanities in Jena, 23.11.2017 [Tagungsbericht], in: H-Soz-Kult, 27.02.2018, online unter: www.hsozkult.de/conferencereport/id/tagungsberichte-7576 (16.04.2019).

5 Vgl. Fabio CІоттı, Digital literary and cultural studies: the state of the art and perspectives, in: Between IV/8 (2014), online unter: http://ojs.unica.it/index.php/between/article/view/1392/1183 (16.04.2019). 
Bis zu einem gewissen Grad an Feinkörnigkeit kann man so, das geeignete Vokabular vorausgesetzt, tatsächlich digital festhalten, was Brennan so schmerzlich vermisst: humane agency - indem sich etwa der Inhalt traditioneller Forschungsbemühungen, der sich ansonsten beispielsweise in einem Aufsatz niederschlagen würde, in RDF formulieren ließe, und das auf eine Art und Weise, die die schon erwähnten Einschränkungen traditioneller Publikationsformen überwindet.

II.

Intertextualität und Semantic-Web/Linked-Data-Technologie scheinen wie füreinander gemacht. So wurde schon früh auf die konzeptionelle Ähnlichkeit zwischen beiden hingewiesen, ${ }^{6}$ insofern als Intertextualitätsforschung ja auch darin besteht, Relationen zwischen Entitäten zu klassifizieren; der prototypische Befund verbindet Text mit Text, also Subjekt über Relation mit Objekt. Dieser Dreischritt entspricht dem RDF-Triple. ${ }^{7}$

Die Modellierung von Intertextualität ist zudem aus zwei ganz praktischen Gründen sehr reizvoll: Der Gegenstandsbereich selbst ist entweder schon online verfügbar oder kann ohne weiteres digitalisiert werden; URI-identifizierte Ressourcen liegen zumindest im Fall bibliographischer Metadaten schon vor. ${ }^{8}$ Zudem deckt der Begriff der Intertextualität, so er voll ausgeschöpft wird, eine überraschend große Bandbreite literaturwissenschaftlicher Tätigkeiten ab. Literaturwissenschaft, die etwa Motivgeschichte, lyrische Formen, kulturelle Kontexte oder Gattungszugehörigkeit behandelt, stellt in jedem dieser Arbeitsbereiche Verknüpfungen her: zwischen Einzeltexten, zwischen Elementen innerhalb eines Textes, zwischen Einzeltexten und abstrakten Konzepten wie Gattungen oder formalen, semantischen oder rhetorischen Merkmalen. Und alles das liegt - je nach Theorie-Frame - entweder ohnehin als Text vor oder hat textuelle Grundlagen oder Repräsentanten.

Dazu kommt eine Eigenschaft, die ich behelfshalber den ,immanenten Konstruktivismus' dieser Technologie nenne (Vorschläge für eine elegantere Formulierung sind willkommen): Das Semantic Web erlaubt, Daten aus der Perspektive unterschiedlichster epistemologischer Positionen, auf unterschiedlichsten methodischen und theoretischen Grundlagen in den Blick zu nehmen. Jedes individuelle Element, über das sich Aussagen treffen lassen (technisch gesprochen: jede URI), kann einer theoretisch unbegrenzten Anzahl von Klassen aus einer ebenso unbegrenzten Anzahl von Ontologien zugeordnet werden; die Ontologien sind einsehbar (und im Idealfall gut dokumentiert), jede Aussage wird so als Interpretation erkennbar, die auf ihre eigenen Voraussetzungen zurückverweist.

Dieser für die Geisteswissenschaften so angemessen erscheinende Rahmen stellt zugleich eine Herausforderung für die Konzeption einer entsprechenden Ontologie dar: Ziel muss es ja sein, dieses Merkmal des Semantic Web auf Ebene des angestrebten Vokabulars wiederherzustellen, um es für die Beherbergung unterschiedlichster Theorieframes tauglich zu machen. Und wenn schon die bereits angesprochene Bandbreite an Tätigkeitsfeldern, in denen im weiteren Sinne intertextuell verfahren

6 Vgl. Joseph F. TEnNIS, URIs and Intertextuality: Incumbent Philosophical Commitments in the Development of the Semantic Web, in: Knowledge Organization and the Global Information Society: Proceedings of the Eighth International ISKO Conference, Würzburg 2004, 103-108.

7 Mit CiTO liegt eine Ontologie vor, die Intertextualität auf diese Art abbilden kann: Silvio PERONI / David SHOTTON, The SPAR Ontologies. Submitted to the 17th International Semantic Web Conference, 2018, online unter: https://w3id.org/spar/article/spar-iswc2018/ (11.05.2018). Die Ontologie ist auf die Abbildung von Zitationsverhältnissen im wissenschaftlichen Diskurs ausgerichtet und damit natürlich hinsichtlich ihrer Anwendbarkeit für die Literaturwissenschaft beschränkt.

8 Zu nennen ist hier natürlich die GND, die „Gemeinsame Normdatei“ der Deutschen Nationalbibliothek (http://www.dnb. de/gnd). 
wird, groß ist, ist die Zahl an unterschiedlichen Möglichkeiten, Intertextualität theoretisch zu konzipieren, noch ungleich größer.

Es geht also darum, Universalität und Domänenspezifik zu versöhnen, das heißt in diesem Fall: eine Ontologie zu konzipieren, die einerseits die groß Bandbreite intertextueller Typen und Theorien umfasst, ohne, dass dabei andererseits die verschiedenen Theoriegebäude, auf denen die zu modellierenden Aussagen beruhen, miteinander in Konflikt geraten.

\section{III.}

Intertextualität, d. h. die Beziehung zwischen Texten, lässt sich theoretisch ganz unterschiedlich fassen, davon zeugen Ansätze, die seit 1968 unter diesem von Julia Kristeva ${ }^{9}$ geprägten Begriff kursieren sowie schon davor unter verschiedenen der Rhetorik oder der Gattungstheorie entstammenden Termini. Zwei Positionen an weit entfernten Polen des breiten Theorie-Spektrums wären etwa die der Einflussforschung auf der einen und die der Intertextualität nach Kristeva auf der anderen Seite. Die Einflussforschung interpoliert aus den Verhältnissen zweier zeitlich aufeinanderfolgender Texte ein Kausalverhältnis, das so aussieht: Ein Autor liest den Text eines anderen Autors, was wiederum seinen eigenen Schreibakt und den resultierenden Text beeinflusst. Kristeva hingegen konzipiert einen zeitund ortlosen Raum, in dem die Gesamtheit aller Texte zueinander in einem Verhältnis gegenseitiger „Absorption und Transformation"10 steht.

Schon an diesen sehr rudimentären Beschreibungen der Theoriegebäude wird deutlich, dass eine Modellierung dieser beiden Typen von Intertextualität Klassen und Relationen verlangen würde, die nicht nur unterschiedlich, sondern tatsächlich inkompatibel wären: In Kristevas Konzeption ${ }^{11}$ gibt es etwa nichts, das sinnvoll der Klasse „Ereignis“ (an einem Punkt in Zeit und Raum) zuzuordnen wäre, während hingegen (biographische, historische) „Ereignisse“ im Bereich der Einflussforschung für die Text-Text-Relation unabdingbar sind.

Die Lösung des Problems hat zwei Seiten: Sinnvoller Ausgangspunkt ist erstens der kleinste gemeinsame Nenner literaturwissenschaftlicher Arbeit. Dieser Nenner ist schlicht die Arbeit am Text. Der kleinste gemeinsame Nenner der Intertextualitätsforschung wiederum ist die Text-Text-Beziehung. Als zentrales Theorem einer entsprechenden Ontologie (und erste Hälfte der Lösung des Problems) bietet sich also der Textualismus an: insofern, als er den Gesprächsgegenstand sinnvoll auf Texte (sowie Textmerkmale) und Relationen reduziert. Andere Aspekte, die je nach Theoriegrundlage ebenso wichtig sein können, wie Autorschaft und Einfluss, Handlungen, Intentionen, Kultur, Systeme, Institutionen etc. stehen dem möglichst universellen Zuschnitt des Vokabulars im Wege. Gerade in seiner scheinbaren Beschränkung bietet der Textualismus das ideale Meta-Modell zur Integration verschiedenster Forschungsmethoden.

9 Julia KRISTEVA, Bachtin, das Wort, der Dialog und der Roman, in: Dorothee Kimmich / Rolf Günter Renner / Bernd Stiegler, Hg., Texte zur Literaturtheorie der Gegenwart, Stuttgart 2004, 334-348.

10 Ebd., 337.

11 Die jedoch gerade durch ihre eigenen Fallstudien nicht exemplifiziert werden - vgl. dazu Jonathan CuLLER, Presupposition and Intertextuality, in: MLN 91/6 (1976), 1380-1396, insbes. 1384f. 
Diese lassen sich dann gewissermaßen indirekt einführen: Indem verschiedene Arten intertextueller Verhältnisse als je unterschiedliche „Typen intertextueller Relation“ formuliert werden: Alles, was über die Text-Text-Relation hinaus theoriespezifisch ist (etwa Ereignisse, Intentionen etc.), ist diesem „Typ“ implizit. ${ }^{12}$

\section{IV.}

Damit sind zwei grundlegende Bausteine für eine Ontologie benannt, mit der man über Texte, Textmerkmale und, darauf aufbauend, über intertextuelle Relationen sprechen können soll: der Textualismus einerseits sowie die Implizitheit methodisch-theoretischer Spezifika andererseits.

Man könnte sich abschließend fragen, ob eine Ontologie, deren Konzeption die eben besprochenen Punkte beachtet, den Forderungen entspricht, die eingangs von Burdick und Brennan aufgestellt wurden. Die Antwort wäre hinsichtlich Brennans impliziter Forderung nach der Abbildung menschlicher agency recht eindeutig: Diesem Anspruch wird eine Ontologie wohl kaum gerecht, die im „Typ intertextueller Relation“ die theoretischen Implikationen - den Kern menschlicher Agency, des Denkens! - auf eine einzige Instanz komprimiert (deren Details sich dann ggf. nur in einem Literal nachlesen lassen).

Resultiert darüber hinaus die Übertragung von Forschungsergebnissen in Linked Data nicht in einem bloßen ,Update des Wissens-Übertragungssystems' - also in dem, was Burdick et al. explizit aus den ,eigentlichen' Digital Humanities ausgeschlossen hatten? Das hängt natürlich vom konkreten Einsatz des jeweiligen RDF-Datensatzes $a b ;{ }^{13}$ man muss aber wohl die Möglichkeit in Betracht ziehen, dass man mit Linked Data-Einsatz keiner Seite der Alternative Burdick-Brennan entspricht und darin dennoch enormes Potenzial für die digitale Modellierung geistes- und literaturwissenschaftlicher Forschung liegt.

\section{Literaturverzeichnis}

Timothy BrennAN, The Digital-Humanities Bust. After a decade of investment and hype, what has the field accomplished? Not much, in: The Chronicle of Higher Education, 15.10.2017.

Ann Burdick u. a., Digital_Humanities, Cambridge (Mass.) / London 2012.

Fabio CıоттI, Digital literary and cultural studies: the state of the art and perspectives, in: Between IV/8 (2014), online unter: http://ojs.unica.it/index.php/between/article/view/1392/1183 (16.04.2019).

Jonathan CULLER, Presupposition and Intertextuality, in: MLN 91/6 (1976), 1380-1396.

Julia KRISTEVA, Bachtin, das Wort, der Dialog und der Roman, in: Dorothee Kimmich / Rolf Günter Renner / Bernd Stiegler, Hg., Texte zur Literaturtheorie der Gegenwart, Stuttgart 2004, 334-348.

Patrick Le Boeuf u. a., Hg., Definition of the CIDOC Conceptual Reference Model. Version 6.2, 2015, 27, online unter: http://www.cidoc-crm.org/sites/default/files/cidoc_crm_version_6.2.pdf (22.4.2019).

12 Ohne an dieser Stelle auf die verschiedenen Alignments von INTRO einzugehen, sei angemerkt, dass CIDOC-CRM, die top-level-Ontologie, an die INTRO anschließt, eine Klasse für diesen genau Zweck bereithält: „E55 Type is the CRM's interface to domain specific ontologies and thesauri. These can be represented in the CRM as subclasses of E55 Type, forming hierarchies of terms [...]." - Patrick Le BoEUf u. a., Hg., Definition of the CIDOC Conceptual Reference Model. Version 6.2, 2015, 27, online unter: http://www.cidoc-crm.org/sites/default/files/cidoc_crm_version_6.2.pdf (22.4.2019).

13 D.h. davon, ob der jeweilige Datensatz bloß zur Speicherung, Dissemination, Visualisierung von Datensätzen als RDF modelliert wird oder auch zum automatisierten Erschließen neuer Daten aus den vorhandenen, zur automatisierten Integration mit anderen Datensätzen etc. 
Bernhard OBERREITHER, INTRO. An Intertextual Relationships Ontology for literary studies, online unter: https:// github.com/BOberreither/INTRO (16.04.2019).

Silvio Peroni / David Shotton, The SPAR Ontologies. Submitted to the 17th International Semantic Web Conference, 2018, online unter: https://w3id.org/spar/article/spar-iswc2018/ (11.05.2018).

Torsten ScHRADE, "Set your data free“ - Vom geisteswissenschaftlichen Datensilo zur vernetzten Wissensbasis mittels Linked Open Data. Siehe Martin PRELL, Digital Humanities in Jena, 23.11.2017 [Tagungsbericht], in: H-Soz-Kult, 27.2.2018, online unter: www.hsozkult.de/conferencereport/id/tagungsberichte-7576 (16.04.2019).

Joseph F. TenNIS, URIs and Intertextuality: Incumbent Philosophical Commitments in the Development of the Semantic Web, in: Knowledge Organization and the Global Information Society: Proceedings of the Eighth International ISKO Conference, Würzburg 2004, 103-108. 